\title{
Un algoritmo genético con fertilización in-vitro aplicado a la sintonización de un regulador difuso
}

\author{
J. Fernando García-Mejía ${ }^{1,2}$, Luis Antonio Gonzalez ${ }^{2}$, Carlos E. Torres-Reyes ${ }^{1}$, \\ J. Arturo Pérez Martínez ${ }^{1}$, J. Antonio Garcia-Mejía ${ }^{1}$ \\ ${ }^{1}$ Universidad Autónoma del Estado de México, Centro Universitario UAEM Atlacomulco, \\ Atlacomulco, Estado de México, México \\ ${ }^{2}$ Universidad Centro Panamericano de Estudios Superiores, \\ Zitácuaro, Michoacán, México \\ \{fgarciam, cetorresr, japerezm, jagarciame\}@uaemex.mx, \\ lage49@yahoo.es
}

\begin{abstract}
Resumen. La teoría de la evolución de las especies formulada por Charles Darwin y las leyes de la herencia descritas por Gregory Mendel sirvieron de andamiaje teórico para que John Holland desarrollara uno de los primeros algoritmos evolutivos para solución de problemas de optimización: el Algoritmo Genético, el cual ha servido como base para la creación de nuevas estrategias evolutivas que tienen la finalidad de reducir tiempos de convergencia y estabilidad, es decir la repetitividad de los resultados con respecto al número de ejecuciones. En este trabajo se propone un algoritmo denominado Algoritmo Genético con Fertilización In Vitro, que tiene como base el Algoritmo Genético simple y los principios de reproducción asistida descritos por Robert Edwards, como medio de optimización de un problema mono objetivo de 19 variables correspondiente a la sintonización de funciones de membresía de un regulador difuso del tipo Mamdani aplicado a una celda termoeléctrica. Los resultados obtenidos son analizados por medio de tests estadísticos, con lo cual se confirma que el algoritmo propuesto tiene mejores resultados y es diferente a un Algoritmo Genético.
\end{abstract}

Palabras clave: algoritmo genético, sintonización de funciones de membresía, algoritmo genético con fertilización in vitro.

\section{In Vitro Fertilization Genetic Algorithm Applied to the Tuning of a Fuzzy Regulator}

\begin{abstract}
The theory of species evolution formulated by Charles Darwin and the laws of inheritance described by Gregory Mendel served as theoretical scaffolding for John Holland to develop one of the first evolutionary algorithms for solving problems of optimization: the genetic algorithm, which has served as a basis for the creation of new evolutionary strategies that have the purpose of reducing times of convergence and stability, namely the repeatability of the results with respect to the number of executions. In this paper an algorithm called In Vitro Fertilization Genetic Algorithm is proposed, which is based on the simple Genetic Algorithm and the principles of assisted reproduction described by Robert Edwards, as a means of optimization of a mono objective problem of 19 variables corresponding to the tuning of membership functions of a Mamdani
\end{abstract}


type fuzzy regulator applied to a thermoelectric cell. The results obtained are analyzed by means of statistical tests, which confirms that the proposed algorithm has better results and is different from a Genetic Algorithm.

Keywords: genetic algorithm, tuning of membership functions, in vitro fertilization genetic algorithm.

\section{Introducción}

Los algoritmos evolutivos, (Evolutionary Algorithms, o EA, por sus siglas en inglés) se pueden definir como una colección de métodos heurísticos, es decir reglas obtenidas de un conocimiento previo de cierto dominio de un problema [1], que tienen pasos definidos inspirados en la mayoría de los casos por procesos biológicos como la reproducción y mutación de los seres vivos, la recombinación y la selección biológica [2] los cuales fueron propuestos por Charles Darwin en el libro "El origen de las especies" [3].

En síntesis, un EA son pasos definidos con la capacidad de evolucionar, los cuales se aplican en la optimización. Estos tienen tres características principales

- Tienen una población. Un EA mantiene un conjunto de soluciones, llamado población, representada por números binarios, en trabajos recientes suele usarse la codificación por números reales, para optimizar o aprender el problema en forma paralela. Este es un principio básico del proceso evolutivo.

- Tienen una función objetivo. Esta es una expresión matemática que suele modelar el problema de optimización. El número de sus variables determina el cómo se genera la población, cuyos elementos son evaluados en esta.

- Operadores de recombinación. Es un conjunto de operaciones aplicadas a los elementos de la población, esto permite producir variaciones en su código, lo cual repercute en la exploración del espacio de soluciones.

Los EA funcionan a partir de una colección de posibles individuos que pueden ser representados por medio del vector $\mathrm{P}(\mathrm{t})=\left\{\mathrm{x}_{1}^{\mathrm{t}}, \mathrm{x}_{2}^{\mathrm{t}}, \ldots, \mathrm{x}_{\mathrm{n}}^{\mathrm{t}}\right\}$ que se denominan población, cada elemento $\mathrm{P}(\mathrm{t})$ representa una posible solución factible a un problema de optimización numérica o combinatoria.

Esta población se somete a operaciones de recombinación y trasformación, posteriormente a un proceso de selección, que se realiza de manera iterativa [4], después de un cierto número de iteraciones se espera que el mejor individuo (aquel que genere el valor más alto de la función de aptitud) converja a un determinado punto que será considerado la solución a un problema de optimización [5].

Los EA se caracterizan por partir de un conjunto de soluciones iniciales que se van transformando por la acción de un conjunto de operadores, los cuales se encargan de "refinar" a las soluciones hasta que estas converjan a un determinado punto denominado solución. La estructura de un algoritmo evolutivo se muestra en la Fig 1, la cual permite observar el carácter iterativo del algoritmo [6]. 


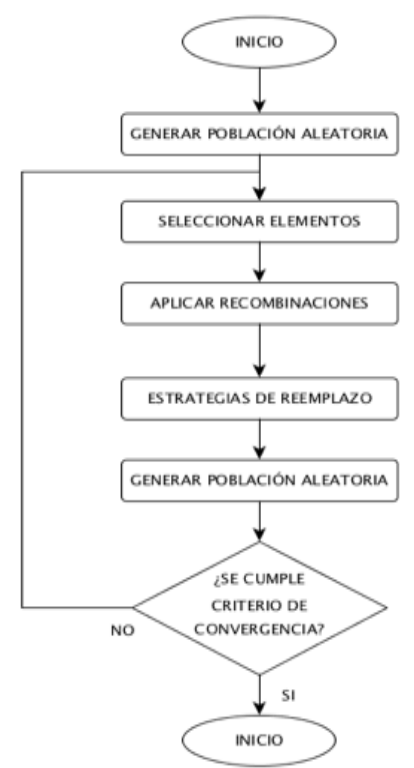

Fig. 1. Esquema genérico de un algoritmo evolutivo basado en los principios biológicos descritos por Darwin y Mendel.

Los algoritmos evolutivos son un área de las tecnologías de la información que continúa en desarrollo, esto es justificable por el teorema "No Free Lunch" desarrollado por David Wolpert y William Macready [7], el cual determina en términos generales que no existe un algoritmo universal de solución de problemas de optimización. Es decir, una técnica que fue exitosa en un entorno no necesariamente funcionará de igual forma en otra situación [8].

\subsection{Algoritmo genético}

John Holland de la Universidad de Michigan en la década de los 60's desarrollo el algoritmo evolutivo por excelencia, el Algoritmo Genético [9] con inspiración en los trabajos sobre la evolución de las especies desarrollados por Charles Darwin [10] (Wang, 2009) donde se estipulan las condiciones de supervivencia del individuo más apto en un entorno. Otro referente teórico en el desarrollo de los GA son las leyes de la herencia desarrolladas por Gregory Mendel [11].

Las ventajas del uso de Algoritmos Genéticos sobre otras técnicas evolutivas son las siguientes $[12,13,14]$.

- Encuentran aproximaciones a problemas sin soluciones prácticas.

- Ideales a problemas complejos de índole adaptativo.

- Habilidad de manipulación para muchos parámetros simultáneamente, dado que los problemas reales no son definibles en términos de una sola variable.

Para su implementación no es necesario poseer un conocimiento a fondo de los problemas que se intentan resolver, solo se realizan cambios aleatorios en las posibles soluciones, evaluándose con la función objetivo para verificar si estos llegan a una mejora. 
Algunas limitaciones y desventajas de los algoritmos genéticos con respecto a otras técnicas evolutivas son $[12,13,14]$.

- Una incorrecta selección de la función objetivo repercute en la capacidad del algoritmo para encontrar la solución correcta del problema.

- Se debe delimitar el tamaño de la población, ritmo de la mutación y cruzamiento ya que, si la población es pequeña el algoritmo puede no explorar todo el espacio de soluciones.

- Que el algoritmo puede converger de forma prematura, si la solución óptima aparece demasiado pronto, mermando las soluciones y llegando a un óptimo local en lugar de explorar todo el espacio y llegar al óptimo global.

- No se deben de utilizar en problemas de solución analítica, ya que los métodos analíticos tradicionales consumen mucho menos tiempo y potencia de la computadora que los algoritmos genéticos.

La Fig. 2 muestra el diagrama de flujo de un Algoritmo Genético simple o Canónico, para la solución de un problema de optimización mono objetivo:

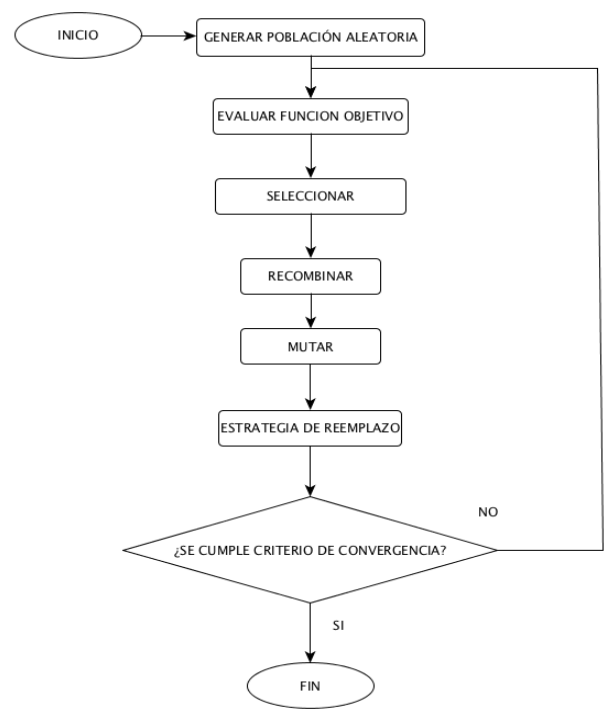

Fig. 2. Diagrama de flujo de un Algoritmo Genético Canónico propuesto por John Holland.

\subsection{Algoritmo genético con fertilización in vitro}

Como se describió en las secciones anteriores un Algoritmo Genético simple es una técnica útil en la solución de problemas de optimización, tanto numéricos como combinatorios, es el algoritmo evolutivo por antonomasia y por lo tanto objeto de estudio continuo en las ciencias computacionales. Un ejemplo de esto es el artículo In Vitro Fertilization Genetic Algorithm Applied To Multidimensional 0-1 Knapsack Problem, desarrollado por Camilo Jr., profesor de la Universidad Federal de Gois [23].

Camilo Jr. propone una variante del GA denominándolo Algoritmo Genético con Fertilización In Vitro IVF/GA (In Vitro Fertilization/ Genetic Algorithm, IVF/GA, por 
sus siglas en inglés) que soluciona los problemas de convergencia lenta de los GA a través de la emulación computacional de un proceso biológico denominado Fertilización In Vitro, una técnica que empezó a ser desarrollada en la década de los 60's del siglo XX por Robert Edwards (Premio Nobel de Medicina en 2010) a partir de la fecundación en un laboratorio con condiciones controladas de óvulos de ratón para posteriormente ser implantados en una hembra de la misma especie.

Las investigaciones realizadas por Edwards, fundamentaron el diseño de un nuevo método heurístico denominado IVF/GA realizado por Camilo Jr. En esta nueva técnica se explora la posibilidad de usar los cromosomas que no fueron seleccionados para recombinación por medio de analogías con el proceso de reproducción asistida de Fertilización in Vitro. De manera original la técnica se basó en la codificación binaria de los cromosomas que forman la población del algoritmo.

La propuesta que se presenta en este trabajo explora un área de oportunidad no explorada de manera original en el trabajo de Camilo, la cual consiste en cambiar las cadenas binarias por vectores $\overrightarrow{\mathrm{x}} \in \mathbb{R}$. Esto permite solucionar problemas de optimización numérica de manera más eficiente con respecto a codificaciones binarias donde los genes $g \in \mathbb{Z}$. La Fig. 3 muestra el diagrama de flujo de un Algoritmo Genético con Fertilización In Vitro y resalta el módulo In Vitro.

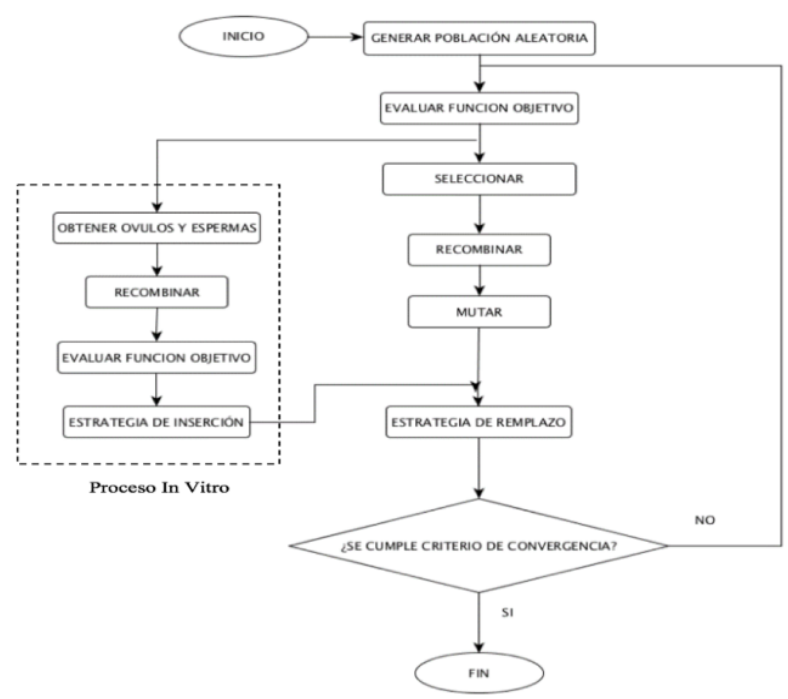

Fig. 3. Algoritmo Genético con Fertilización In Vitro, este módulo se observa resaltado por medio de línea punteada.

\section{Caso de prueba}

Las Celdas Termoeléctricas (Thermo Electric Cooler, TEC, por sus siglas en inglés), son una alternativa usada en la refrigeración móvil, dado que su funcionamiento no depende de gases Hidrofluorocarbonatados, los cuales han sido prohibidos en algunos países por el daño ecológico que causan. Una TEC (cuyo esquema se muestra en la 
Figura 1están constituidas por materiales semiconductores tipo P y N, encapsulados en una superficie cerámica elaborada a partir de óxido de berilio $(\mathrm{BeO})$ mezclado con Alúmina $\left(\mathrm{Al}_{2} \mathrm{O}_{3}\right)$, una alternativa a este es el nitrato de aluminio $\left(\mathrm{Al}\left(\mathrm{NO}_{3}\right)_{3}\right)$ [15].

El fenómeno de transferencia de energía en forma de calor (enfriamiento) puede modelarse por medio de una función de transferencia en términos de la variable compleja s tal como lo muestra la expresión 1 [16]:

$$
\frac{\widetilde{T}_{L}(s)}{\tilde{I}(s)}=G_{I}(s)=-6.4061\left(\frac{0.064 s+0.00854}{s^{2}+0.5964 s+0.00855}\right) .
$$

El comportamiento descrito en la expresión 1 puede modificarse por medio del uso de técnicas de control inteligente como lo muestra [17], el enfriamiento de un diodo de avalancha mediante una TEC, controlada para alcanzar una referencia dada mediante un controlador proporcional difuso de algoritmo de Takagi-Sugeno del tipo Una Entrada, Una Salida (Single Input, Single Output, SISO, por sus siglas en inglés), el cual es simulado en simulink a partir de un modelo matemático linealizado en función de la temperatura y corriente de consumo, consiguiendo la estabilización del mismo a $-19.44^{\circ} \mathrm{C}$ en 35.91 segundos.

El modelo matemático empleado en [18]y [19] solo funciona para una referencia predeterminada y ciertas condiciones iniciales. Con esta finalidad se continúa la línea de trabajo de los controladores PID, combinándolos con redes neuronales artificiales [18] y se propone el uso de un esquema basado en una red neuronal de 3 capas con funciones de activación de base radial. En este desarrollo se utiliza un cuantificador difuso, el cual tiene como función normalizar las salidas del observador mediante el uso de funciones sigmoidales. Teniendo un tiempo de establecimiento para una referencia de -20 grados en 60 segundos. En [19] se controla una TEC mediante un modelo matemático que hace uso de la potencia de disipación de la misma, utilizando un controlador difuso de algoritmo de Mamdani del tipo SISO, con funciones de pertenencia del tipo gaussiana, con lo cual se busca reducir la velocidad de respuesta en los cambios de temperatura.

En [20] se simula un radiador para un nano satélite en el cual uno de sus componentes es una celda TEC la cual es controlada por medio de un algoritmo de Mamdani del tipo MIMO donde las entradas son las temperaturas tanto ambiente como la de la cara fría y las salidas son salidas de corriente, esto tiene como finalidad controlar el calor generado.

En este trabajo se propone el diseño de un regulador difuso aplicado a una TEC a partir de un Algoritmo Genético con Fertilización In Vitro, cuya población es codificada por medio de números reales. Con lo cual se espera mejoras en los tiempos de establecimiento, sobre impulso y valor final del sistema de control, así como mejoría en la estabilidad y convergencia con respecto a un Algoritmo Genético simple.

\section{Metodología}

El esquema metodológico empleado para mostrar la utilidad de un Algoritmo Genético con Fertilización In Vitro con respecto a la versión canónica de este como medio de sintonización de funciones de membresía de un controlador difuso cuyo esquema se muestra en la Fig. 4, en la cual como se puede observar se plantea un 
sistema de Múltiples Entradas- Una Salida (Multiple Inputs Single Output,MISO por sus siglas en inglés) cuyas funciones de membresía y conjuntos difusos para las variables de entrada son definidos por lo mostrado de acuerdo en la tabla 1, para las variables de salida estos parámetros son definidos en la tabla 2.

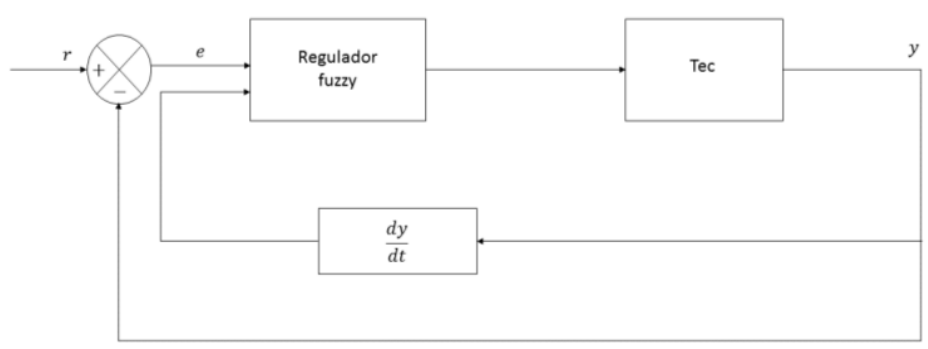

Fig. 4. Diagrama esquemático del regulador difuso propuesto.

Tabla 1. Variable de entrada y sus conjuntos difusos.

\begin{tabular}{lllc}
\hline Variable & \multicolumn{2}{l}{ Conjuntos difusos } & Función de membresía \\
\hline er & ne & Error negativo & $\mu_{\text {sigmoide }}\left(\mathrm{er} ; \mathrm{e}_{1}, \mathrm{e}_{4}\right)$ \\
(error) & $\mathrm{c}$ & Error Cero & $\mu_{\text {gaussbellf }}\left(\mathrm{er} ; \mathrm{e}_{3}, \mathrm{e}_{4}, 0\right)$ \\
& pe & Error Pequeño & $\mu_{\text {sigmoide }}\left(\mathrm{er} ; \mathrm{e}_{5}, \mathrm{e}_{6}\right)$ \\
ds & ns & Incremento & $\mu_{\text {sigmoide }}\left(\mathrm{er} ; \mathrm{e}_{7}, \mathrm{e}_{8}\right)$ \\
(incremento de & & negativo & \\
salida) & nc & Sin incremento & $\mu_{\text {gaussbellf }}\left(\mathrm{er} ; \mathrm{e}_{9}, \mathrm{e}_{10}, \mathrm{e}_{11}\right)$ \\
& $\mathrm{np}$ & Incremento Pequeño & $\mu_{\text {sigmoide }}\left(\mathrm{er} ; \mathrm{e}_{12}, \mathrm{e}_{13}\right)$ \\
\hline
\end{tabular}

Tabla 2. Variable de salida y sus conjuntos difusos (Elaboración propia).

\begin{tabular}{lllc}
\hline Variable & \multicolumn{2}{l}{ Conjuntos difusos } & Función de membrecía \\
\hline pe & pn & Pendiente negativa & $\mu_{\text {sigmoide }}\left(\mathrm{pe} ; \mathrm{e}_{14}, \mathrm{e}_{15}\right)$ \\
(pendiente de & $\mathrm{pc}$ & Pendiente cero & $\mu_{\text {gaussbellf }}\left(\mathrm{er} ; \mathrm{e}_{15}, \mathrm{e}_{16}, \mathrm{e}_{17}\right)$ \\
control & $\mathrm{pp}$ & Pendiente positiva & $\mu_{\text {sigmoide }}\left(\mathrm{er} ; \mathrm{e}_{18}, \mathrm{e}_{19}\right)$ \\
\hline
\end{tabular}

De tal forma que la Fig. 5 muestra la FAM (Memoria Asociativa Difusa), la cual relaciona las variables de entrada con la pendiente de control que se constituye como señal de control para la regulación de la TEC.

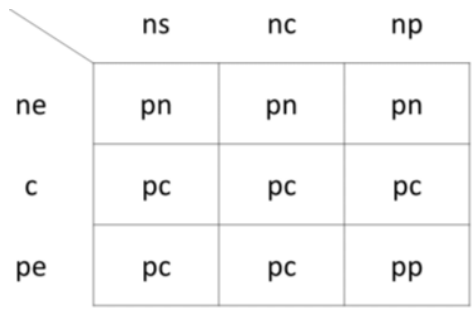

Fig. 5. Reglas de inferencia representadas en una Memoria Asociativa Difusa.

Con base en lo anterior se propone un cromosoma que tiene la forma: 


$$
\text { cromosoma }=\left[\mathrm{e}_{1}, \mathrm{e}_{2}, \ldots \mathrm{e}_{19}\right]^{\mathrm{T}} .
$$

Con una función objetivo que puede describirse como se muestra en la ecuación 2:

$$
\mathrm{f}_{\mathrm{obj}}=\max \left(\frac{1}{\int \frac{1}{\mathrm{~T}}(\text { referencia }- \text { salida })^{2}}\right) .
$$

Después de proponer el esquema del cromosoma y una función objetivo susceptible a ser maximizada, es necesaria la creación de cromosomas, cuyos genes son creados por medio de una distribución de probabilidad uniforme de acuerdo a la tabla 3 :

Tabla 3. Condiciones de generación de cromosomas.

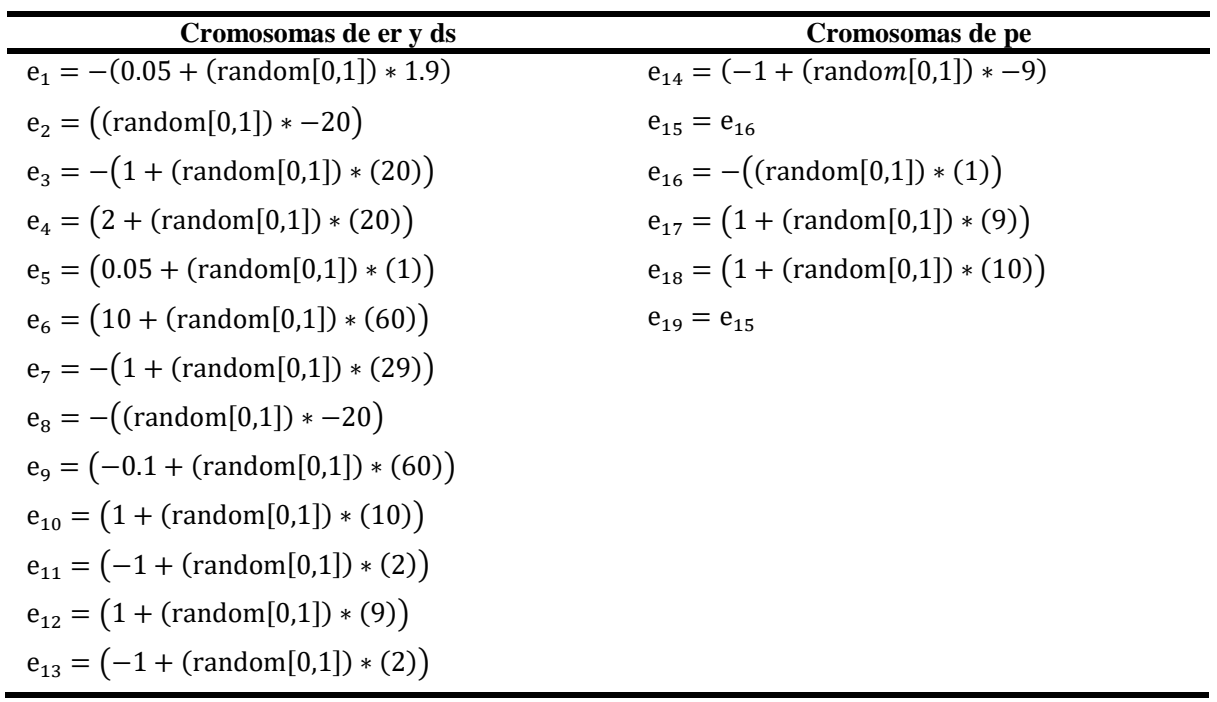

Para probar el efecto del algoritmo IVF/GA se propone un conjunto de experimentos los cuales permiten discernir si existen diferencias estadísticamente significativas de la técnica propuesta con respecto a un GA convencional. La descripción de los experimentos se muestra en la tabla 4.

El operador propuesto para este conjunto de experimentos es el operador de cruzamiento por mezcla (Blend Crossover, BLX- $\alpha$, por sus siglas en inglés) un operador de recombinación que a partir de dos cromosomas progenitores $\mathrm{C}^{\mathrm{H} 1}, \mathrm{C}^{\mathrm{H} 2}$ genera un descendiente de forma aleatoria a la vez de acuerdo a la expresión 3 [21]:

$$
\mathrm{C}^{\mathrm{H}}=\operatorname{rand}\left[\left(\mathrm{h}_{\min }-\mathrm{I} * \alpha\right),\left(\mathrm{h}_{\max }+\mathrm{I} * \alpha\right)\right],
$$

donde $C^{H}$ es el cromosoma descendiente $h_{\min }=\min \left(C_{i}^{1}, C_{i}^{2}\right), h_{\max }=\max \left(C_{i}^{1}, C_{i}^{2}\right)$, $I=h_{\max }-h_{\min }, \alpha=\operatorname{rand}[0,1]$ con distribución uniforme. Cabe destacar que se pueden producir los descendientes que sean necesarios. 
Un algoritmo genético con fertilización in-vitro aplicado a la sintonización de un regulador difuso

Tabla 4. Experimentos propuestos para el regulador difuso.

\begin{tabular}{|c|c|c|c|c|c|c|c|}
\hline No & Técnica & $\begin{array}{c}\text { Porcentaje } \\
\text { de cruza }\end{array}$ & $\begin{array}{c}\text { Porcentaje } \\
\text { de } \\
\text { mutación }\end{array}$ & $\begin{array}{c}\text { Número de } \\
\text { Cromosomas } \\
\text { iniciales }\end{array}$ & $\begin{array}{c}\text { Número } \\
\text { de } \\
\text { óvulos }\end{array}$ & $\begin{array}{c}\text { Número } \\
\text { de } \\
\text { espermas }\end{array}$ & $\begin{array}{c}\text { Número de } \\
\text { gametos } \\
\text { insertados }\end{array}$ \\
\hline 1 & GA & $80 \%$ & $1 \%$ & 60 & - & & - \\
\hline 2 & GA & $60 \%$ & $1 \%$ & 60 & & & \\
\hline 3 & IVF/GA & $80 \%$ & $1 \%$ & 60 & 1 & 24 & 15 \\
\hline 4 & IVF/GA & $60 \%$ & $1 \%$ & 60 & 1 & 24 & 15 \\
\hline
\end{tabular}

El operador de mutación empleado es la mutación Gaussiana es encargado de modificar a un cromosoma específico $\mathrm{C}$ elegido al azar por medio de una distribución de probabilidad gaussina de media 0 y varianza definida como lo muestra la expresión 4 para cada gen $g$ [22]:

$$
\sigma_{\mathrm{k}}=\frac{\mathrm{T}-\mathrm{t}}{\mathrm{T}} \frac{\left(\mathrm{g}_{\mathrm{k}}^{\max }-\mathrm{g}_{\mathrm{k}}^{\min }\right)}{3},
$$

donde t es la generación actual, T es el máximo número de generaciones contempladas en el algoritmo de tal forma que el cromosoma mutado puede definirse como lo muestra 5 .

$$
\mathrm{C}^{\prime}=\mathrm{C}+\mathrm{N}\left(0, \sigma_{\mathrm{k}}\right) \text {. }
$$

\section{Resultados}

En esta sección se muestran los resultados del diseño de funciones de membresía por medio de los algoritmos GA y el IVF/GA. El primer aspecto a verificar es la convergencia, la figura 6 a muestra este criterio para los experimentos 2 y 4 , mientras que la figura $6 \mathrm{~b}$ los muestra para los experimentos 1 y 3 .

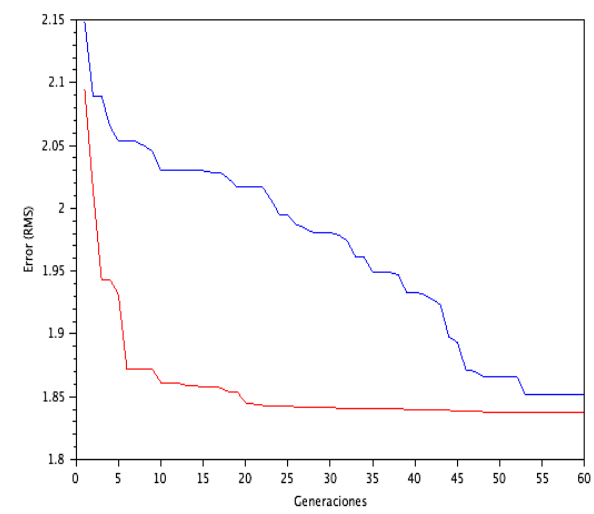

(a)

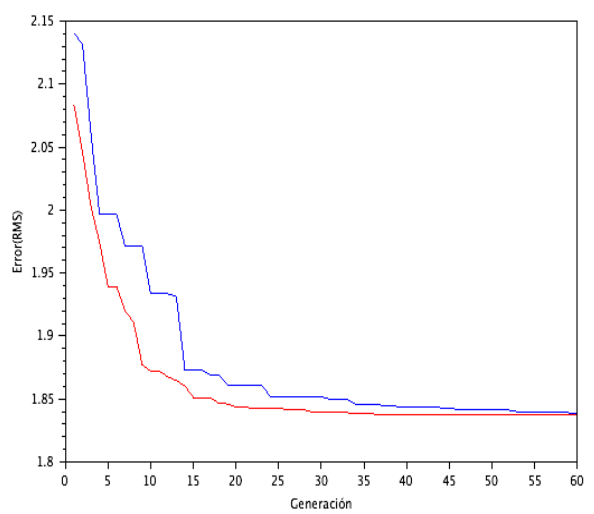

(b)

Fig. 6. Algoritmo Genético con Fertilización In Vitro, este módulo se observa resaltado por medio de línea punteada. 
J. Fernando García-Mejía, Luis Antonio Gonzalez, Carlos E. Torres-Reyes, et al.

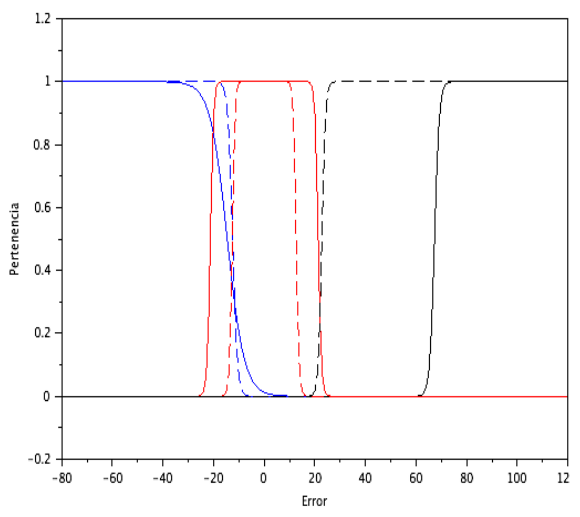

(a)

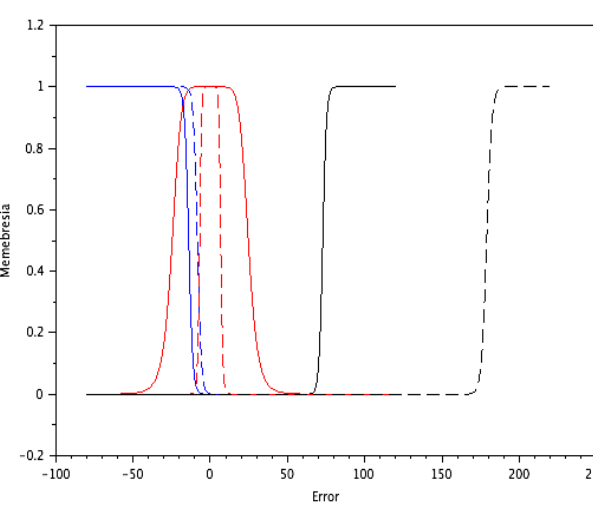

(b)

Fig. 7. Conjuntos difusos variable error GA (azul) e IVF/GA (rojo).

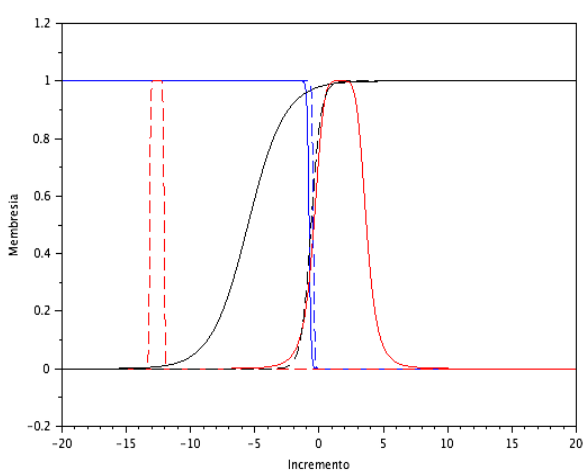

(a)

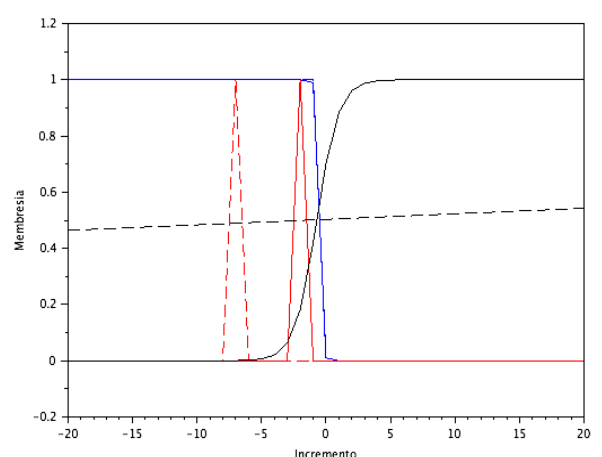

(b)

Fig. 8. Conjuntos difusos variable incremento de salida GA (azul) e IVF/GA (rojo).

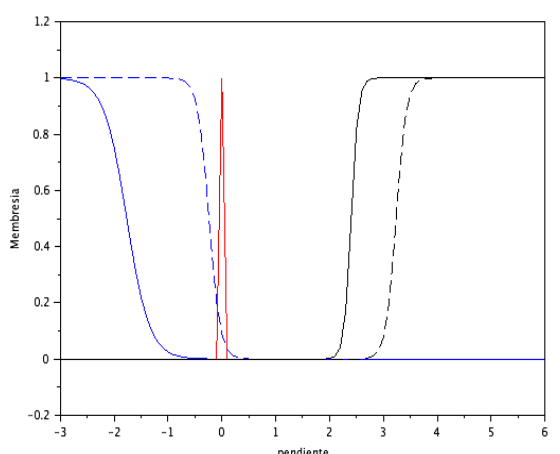

(a)

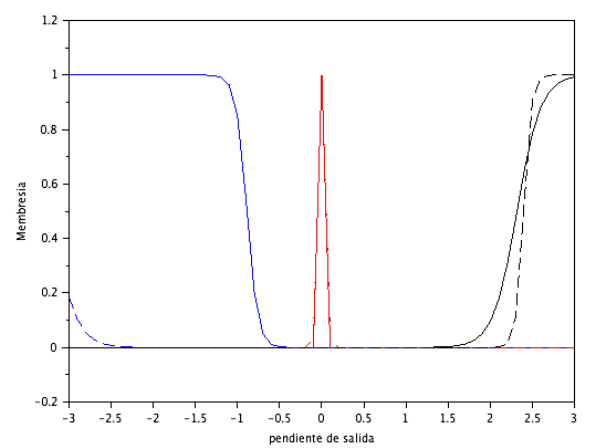

(b)

Fig. 9. Conjuntos difusos variable pendiente de salida GA (azul) e IVF/GA (rojo). 


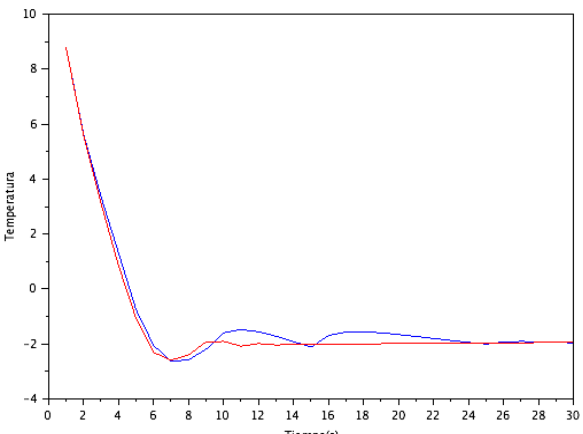

(a)

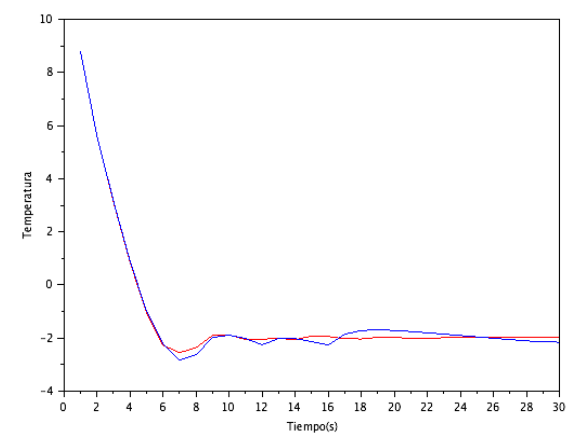

(b)

Fig. 10. Desempeño de los controladores propuestos en este trabajo.

Los conjuntos difusos para la variable error son mostrados en la figura 7a. En color azul, rojo y negro se representan las etiquetas lingüísticas error negativo, error cero y error positivo respectivamente. Cabe destacar que estos fueron ajustados por un GA con un porcentaje de cruza del $60 \%$ que se muestra en línea continua (experimento 2) y en línea punteada el IVF/GA con el mismo porcentaje (experimento 4). Los resultados de los experimentos 1 y 3 se muestran en la figura $7 \mathrm{~b}$.

Los conjuntos difusos para la variable incremento de la salida son mostrados en la Figura 8a. En color azul, rojo y negro se representan las etiquetas lingüísticas incremento negativo, incremento cero e incremento positivo respectivamente. Al igual que en la Figura 7 los conjuntos son ajustados por un GA con un porcentaje de cruza del $60 \%$ que se muestra en línea continua y en línea punteada el IVF/GA con el mismo porcentaje. La figura $8 \mathrm{~b}$ muestra los resultados para los experimentos 1 y 3 .

Los conjuntos difusos para la variable incremento de la salida son mostrados en la Figura 9a. En color azul, rojo y negro se representan las etiquetas lingüísticas incremento negativo, incremento cero e incremento positivo respectivamente. Al igual que en la Figura 7 los conjuntos son ajustados por un GA con un porcentaje de cruza del 60\% que se muestra en línea continua y en línea punteada el IVF/GA con el mismo porcentaje. La figura 9b muestra los resultados para los experimentos 1 y 3 .

La figura 10a muestra la gráfica de salida de temperatura, en el caso de los experimentos 2,4 la 10b indica la salida de los experimentos 2 y 4 . Así mismo la tabla 5 muestra los criterios de desempeño que permite valorar el controlador propuesto.

La tabla 5 muestra los índices de desempeño que permite valorar la acción de los controladores diseñados en esta propuesta. En color rojo se indica cual s el controlador que tiene el mejor desempeño.

Tabla 5. Experimentos para el regulador difuso (Elaboración propia).

\begin{tabular}{|l|l|l|l|l|l|}
\hline \multirow{2}{*}{$\%$ Cruza } & \multicolumn{1}{|c|}{$\begin{array}{c}\text { Técnica } \\
\text { empleada }\end{array}$} & \multicolumn{1}{|c|}{ Error RMS } & $\begin{array}{c}\text { Porcentaje de } \\
\text { sobreimpulso }\end{array}$ & $\begin{array}{c}\text { Tiempo de } \\
\text { establecimiento }\end{array}$ & $\begin{array}{c}\text { Error } \\
\text { estado } \\
\text { estable }\end{array}$ \\
\hline $60 \%$ & GA & 1.8519856 & 31.391 & 25 & -0.0431 \\
\cline { 2 - 6 } & IVF/GA & 1.8374284 & 29.386 & 19 & -0.0589 \\
\hline $80 \%$ & GA & 1.8390043 & 41.3206 & 26 & 0.1550 \\
\cline { 2 - 6 } & IVF & 1.837053 & 27.389 & 22 & -0.0323 \\
\hline
\end{tabular}


Al igual que los experimentos anteriores se procedió a realizar un estudio estadístico con la finalidad de determinar la estabilidad y si existen diferencias estadísticamente significativas entre la aplicación de un GA y el IVF/GA propuesto en la presente disertación doctoral.

En la tabla 6 se muestran los resultados estadísticos realizados sobre 30 ejecuciones (por razones de costo computacional), lo cual permite determinar cuál algoritmo cumple con los mejores criterios de estabilidad a través del cálculo de la repetitividad (desviación estándar entre media aritmética).

Además, se aplicó un test de Kolmogorov-Smirnov (con la finalidad de comprobar el comportamiento estocástico de los algoritmos propuestos).

Tabla 6. Estadísticos de los experimentos sobre el regulador difuso

\begin{tabular}{lrlll}
\hline \multicolumn{1}{c}{ Técnica } & $\begin{array}{r}\text { Valor } \\
\text { Medio }\end{array}$ & $\begin{array}{c}\text { Desviación } \\
\text { Estándar }\end{array}$ & \multicolumn{1}{c}{ Repetitividad } & $\begin{array}{r}\text { Significancia asintótica } \\
(\mathrm{K}-\mathrm{S})\end{array}$ \\
\hline GA 60\% & 1.868189 & 0.028763 & 1.53961 & 0.360 \\
& 1.860960 & 0.034463 & 1.851932 & 0.067 \\
GA 80\% & & & & 0.041 \\
IVF/GA 60\% & 1.853108 & 0.023630 & 1.275155 & 0.047 \\
IVF/GA 80\% & 1.848904 & 0.0236300 & 1.132434 & \\
\hline
\end{tabular}

\section{Conclusiones}

Como se muestra en la tabla 5, la mejor alternativa se constituye por medio del ajuste de funciones objetivos realizados con el IVF/GA con un porcentaje de cruza de $80 \%$. Los estudios estadísticos descritos en la tabla 6 muestra que el Algoritmo Genético tiene un comportamiento que corresponde a una distribución normal, mientras que la propuesta realizada por medio de un IVF/GA no tiene este comportamiento, dado que los valores de significancia de Kolmogorov-Smirnov es menor a un valor de 0.05. Al aplicar el test de Anova sobre los datos obtenidos con el GA se obtiene el siguiente resultado: No existen diferencias estadísticamente significativas al variar los operadores descritos. Por lo tanto, no es necesario aplicar un test post-hoc.

Cuando no existe el comportamiento con distribución normal el test estadístico a usar es Wilcoxon, el resultado de esta prueba arroja una significancia de 0.645 por lo tanto no existen diferencias estadísticas significativas al variar los porcentajes de cruza de un IVF/GA. Esto hace necesario el uso de una prueba post hoc.

Por lo tanto, se asume que se trata de dos algoritmos distintos dado que sus efectos son externamente diferentes. Pero de manera interna no existen diferencias con respecto a cambios en operadores. Esto, en términos de estadística, expresa que si existen diferencias estadísticamente significativas entre sintonizar un controlador Fuzzy con un GA vs IVF/GA. El Algoritmo Genético con Fertilización In Vitro puede sintonizar las funciones de pertenencia de un controlador difuso tal como lo muestran los resultados de las secciones que forman este documento. 


\section{Referencias}

1. Ergun, H., Van Hertem, D.: HVDC Grid Planning. En: Van Hertem, D., Gomis-Bellmunt, O., Liang, J.: HVDC Grids: For offshore and Supergrid of the Future, pp. 143-172. New Jersey, Estados Unidos: IEEE Press (2016)

2. Chacón Montes, P.: Algoritmos Evolutivos: algoritmos genéticos y de cuasiespecie. En I. Ramos Salavert, Vida Artificial, pp. 87-111. Castilla, España: Universidad de Castilla-La Mancha (1995)

3. Vasiljevic, D.: Classical and Evolutionary Algorithms in the Optimization of Optical Systems. New York: Springer Science+Business Media (2002)

4. Robusté Antón, F.: Logística del transporte. Barcelona, España: Edicions de la Universitat Politècnica de Catalunya (2005)

5. Chi, Z., Yan , H., Pham, T.: Fuzzy Algorithms: with Applications to Image Processing and Pattern Recognition. New Jersey, Estados Unidos: World Scientific Publishing (1996)

6. Sumathi, S., Surekha, P.: Computational Intelligence Paradigms: Theory and Applications using Matlab. Boca Raton, Florida, Estados Unidos: CRC Press Taylor \& Francis

7. Group.Ergun, H., Van Hertem, D.: HVDC Grid Planning. En: D. Van Hertem, O. GomisBellmunt, \& J. Liang, HVDC Grids: For offshore and Supergrid of the Future, pp. 143-172. New Jersey, Estados Unidos: IEEE Press (2010)

8. Lloyd, S.: How Smart Is the Universe? En: J. Brockman, Intelligent Thought: Science Versus the Intelligent Design Movement, pp. 179-191. New York, Estados Unidos: Vintage Books (2006)

9. Du, K.-L., Swamy, M.N.: Search and Optimization by Metaheuristics: Techniques and Algorithms Inspired by Nature. Suiza: Springer (2016)

10. Yang, X.-S.: Nature-Inspired Metaheuristic Algorithm. Frome, Reino Unido: Luniver Press (2010)

11. Wang, X., Ruan, D., Kerre, E.E.: Mathematics of Fuzziness - Basic Issues. Berlín, Alemania: Springer (2009)

12. Flake, G.W.: The Computational Beauty of Nature: Computer Explorations of Fractals, Chaos, Complex Systems, and Adaptation. Cambridge, Massachusetts, Estados Unidos: MIT Press (1998)

13. Munakata, T.: Fundamentals of the New Artificial Intelligence: Neural, Evolutionary, Fuzzy and More. Londres, Reino Unido: Springer (2008)

14. Rajagopal, K.: Operations Research. Nueva Delhi, India: PHI Learning Pvt (2012)

15. Carter, J.N.: Introduction to Using Genetic Algorithms. In: Nikravesh, M., Aminzadeh, F., Zadeh, L. A.: Soft Computing and Intelligent Data Analysis in Oil Exploration, pp. 51-76. Amsterdam, Holanda: Elsevier (2003)

16. Garimella, S.V., Harirchian, T.: Encyclopedia of Thermal Packaging: Thermal Packaging Techniques, vol. 1. (A. Bar-Cohen, Ed.) Singapur, Singapur: World Scientific (2013)

17. Shaojing, S., Qin, Q.: Temperature Control of Thermoelectric Cooler Based on Adaptive NN-PID. In: 2010 International Conference on Electrical and Control Engineering pp. 22452248. Wuhan: IEEE. doi:10.11019/iCECE.2010.553 (2010)

18. Sumathi, S., Hamsapriya, T., Surekha, P.: Evolutionary Intelligence: An Introduction to Theory and Applications with Matlab. Berlín, Alemania: Springer (2008)

19. Zang, H.-Q., Li, Q.: The automatic temperature system with fuzzy self-adaptive PID control in semiconductor laser. In: 2009 IEEE International Conference on Automation and Logistics, pp. 1691-1694. Shenyang: IEEE. doi:10.1109/ICAL.2009.5262694 (2009)

20. Aly, A.A., Abo El-Lail, A.S.: Fuzzy Temperature Control of a Thermoelectric Cooler. In: 2006 IEEE International Conference on Industrial Technology, pp. 1580-1585. Mumbai: IEEE. doi:10.1109/ICIT.2006.372492 (2006) 
J. Fernando García-Mejía, Luis Antonio Gonzalez, Carlos E. Torres-Reyes, et al.

21. Li, Y.-Z., Lee, K.-M., Wang, J.: Analysis and Control of Equivalent Physical Simulator for Nanosatellite Space Radiator. IEEE/ASME Transactions on Mechatronics, 15, pp. 79-87. doi:10.1109/TMECH.2009.2016957 (2010)

22. Bakshi, U.A., Bakshi, V.U.: Automatic Control System. Pune, India: Technical Publications Pune (2008)

23. Bansal, K.: Manual of Intrauterine Insemination (IUI), In Vitro Fertilization (IVF) and Intracytoplasmic Sperm Injection (ICSI). Nueva Delhi, India: Jaypee Brothers Medical Publishers (2011)

24. Camilo, C., Yamanaka, K.: In Vitro Fertilization Genetic Algorithm Applied to Multidimensional 0-1 Knapsack Problem. In: The 2011 World Congress in Computer Science, Computer Engineering and Applied Computing. Las Vegas: World Academy of Sciences (2011) 